\title{
Traumatic synovitis in a classical guitarist: a study of joint laxity
}

\author{
H. A. BIRD AND V. WRIGHT
}

From the Rheumatism Research Unit, University Department of Medicine, General Infirmary at Leeds, and Royal Bath Hospital, Harrogate

SUMMARY A classical guitarist performing for at least 5 hours each day developed a traumatic synovitis at the left wrist joint that was first erroneously considered to be rheumatoid arthritis. Comparison with members of the same guitar class suggested that unusual joint laxity of the fingers and wrist, probably inherited from the patient's father, was of more importance in the aetiology of the synovitis than a wide range of movement acquired by regular practice. Hyperextension of the metacarpophalangeal joint of the left index finger, quantified by the hyperextensometer, was less marked in the guitarists than in 100 normal individuals. This may be attributed to greater muscular control of the fingers. Lateral instability in the loaded joint may be the most important factor in the aetiology of traumatic synovitis.

Joint laxity at the hands may be advantageous for musicians. The violinist Paganini was said to display abnormal laxity with long thin fingers, which accounts for the technical difficulty of the compositions of this composer and performer. ${ }^{1} \mathrm{Ab}$ normal joint laxity can predispose to a synovitis, presumed traumatic, that mimics rheumatoid arthritis. ${ }^{2}$ We here describe the case of a classical guitarist who presented with an erroneous diagnosis of rheumatoid arthritis and the subsequent studies we performed to assess the laxity of the hands in other guitarists in the same class and in the normal population.

\section{Subjects and methods}

\section{CASE HISTORY}

A 31-year-old male was a student of the classical guitar. For 10 years he had practised up to 5 hours each day and was of advanced performer standard. In June 1978 he noticed pain at the back of the left wrist associated with swelling and exacerbated by practising the guitar. It was not present in the other hand and no other joints were involved. In December 1978 he sought the advice of his general practitioner, and a cystic swelling of the synovium on the dorsum of the left wrist was observed. This particular hand was subjected to considerable stretching in the course of his playing (Fig. 1). He considered himself double-jointed in comparison with other members of the class (Fig. 2). Investigations included an erythrocyte sedimentation rate (ESR) of $1 \mathrm{~mm} \mathrm{~h}^{-1}$ and normal $x$-rays of hands and wrists.

In May 1979 he was seen in the Rheumatology Clinic of the Leeds General Infirmary. The swelling of the synovium on the dorsal aspect of the left wrist had persisted, his symptoms being controlled

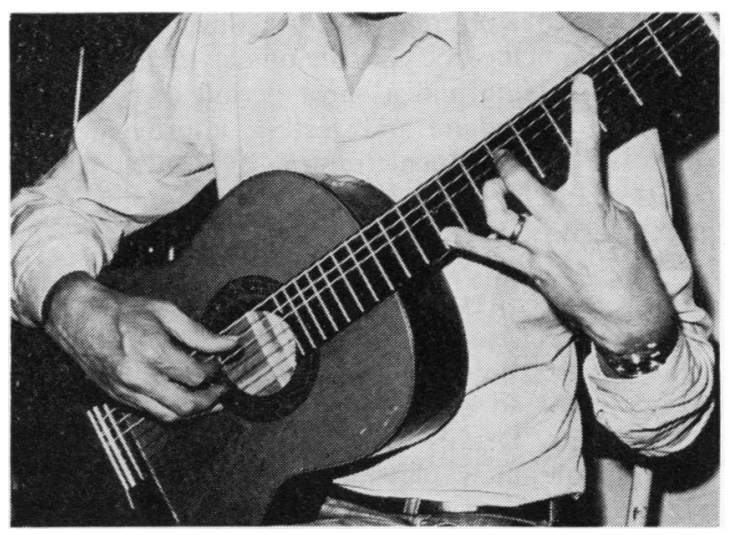

Fig. 1 The position of the left hand adopted by the patient in playing the classical guitar. The traumatic synovitis of the wrist joint is clearly visible. The right hand is not subjected to this degree of stretching. 


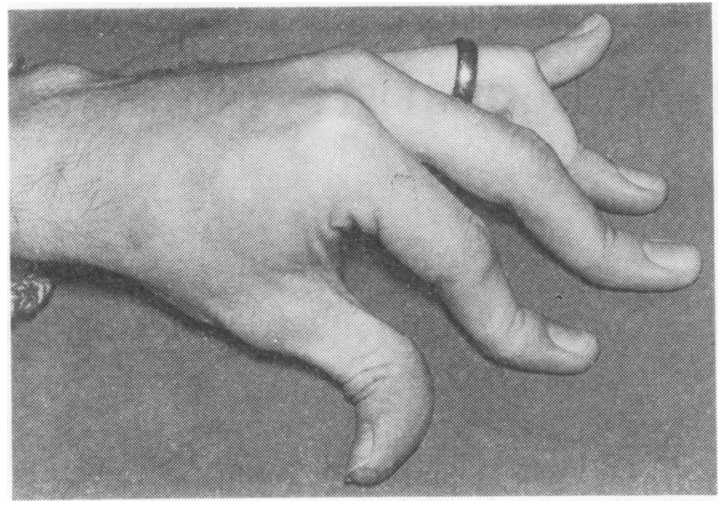

Fig. 2 The unusual degree of joint laxity of the patient's hands

on indomethacin $25 \mathrm{mg}$ t.d.s. There was no early morning stiffness and, apart from possible intermittent swelling of some proximal interphalangeal joints, no other symptoms. His father had considered himself double-jointed and a maternal aunt and grandmother were both said to have had rheumatoid arthritis. There was an unusual degree of laxity in both hands, but this was not so striking elsewhere (Carter and Wilkinson score ${ }^{3}=4 / 9$ ). There was no clinical evidence of rheumatoid disease at the metacarpal or metatarsal heads or at the ulnar styloid. All other joints were normal. The haemoglobin was $15 \cdot 1 \mathrm{~g} / \mathrm{dl}$, white blood count $9 \cdot 1 \times 10^{9} / 1$ and the ESR $5 \mathrm{~mm} /$ hour. A test for antinuclear factor was negative, a latex fixation test for rheumatoid factor was negative, sheep cell agglutination test (SCAT) sensitised cells $1: 4$. $X$-rays of the hands and feet were normal.

The patient was treated with a single injection of $2 \mathrm{mg}$ triamcinolone hexacetonide to the cystic synovial swelling and a short period of rest. This produced immediate and lasting improvement in spite of a subsequent return to regular guitar practice, and he has had no further symptoms in the last 9 months (Fig. 3).

\section{OTHER GUITARISTS AND RANDOM SAMPLE}

The apparent association between the patient's joint laxity, his occupation, and the synovitis prompted us to study the other members of his guitar class. Details were collected of age, sex, duration of guitar playing, hyperextension of the metacarpophalangeal joint of the left index finger measured by the finger hyperextensometer devised at Leeds, ${ }^{4}$ and a visual assessment of lateral laxity of the fingers made by eye and graded as $+1++1++$.

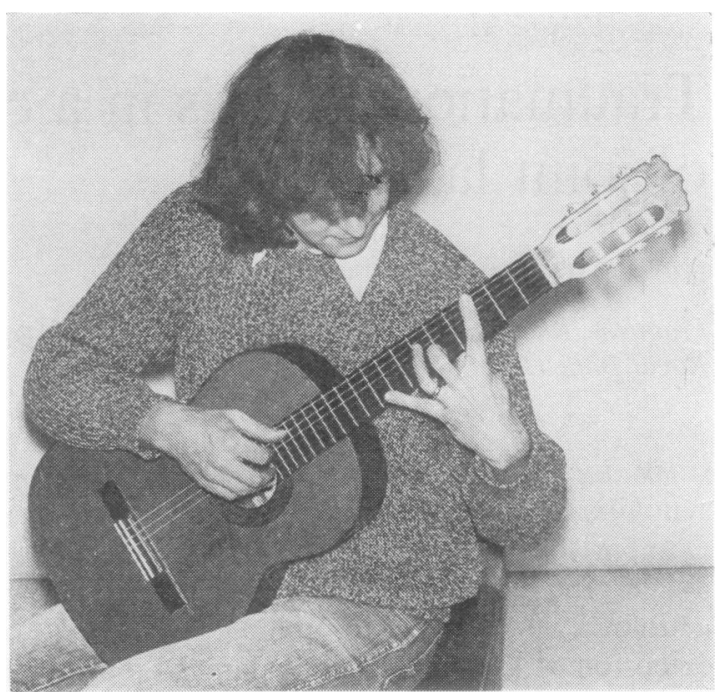

Fig. 3 The guitarist 9 months after treatment with a single injection of triamcinolone hexacetonide to the left wrist. The synovitis has now resolved.

The finger hyperextensometer was also used to assess laxity of the same joint in 100 normal people, drawn at random from a Caucasian population.

\section{Results}

The degree of joint laxity in the guitarists is shown in Table 1. It did not correlate with the duration of guitar playing and the patient with synovitis reported on here had the third highest degree of finger extension in the class in spite of his age. Taken in conjunction with his marked lateral laxity, more pronounced than in any other subject, he could be

Table 1 Details of patient and 13 other members of a classical guitar class

\begin{tabular}{cccclc}
\hline Subject & Sex & Age & $\begin{array}{l}\text { Duration of } \\
\text { guitar } \\
\text { playing } \\
\text { (years) }\end{array}$ & $\begin{array}{l}\text { Hyperextension of } \\
\text { MCP joint of } \\
\text { left index finger } \\
\text { (degrees) }\end{array}$ & $\begin{array}{l}\text { Lateral } \\
\text { laxity } \\
\text { of } \\
\text { fingers }\end{array}$ \\
\hline 1 & M & 39 & 25 & 38 & ++ \\
$2^{*}$ & M & 31 & 12 & 56 & ++ \\
3 & M & 26 & 4 & 58 & + \\
4 & M & 26 & 11 & 12 & + \\
5 & F & 25 & 6 & 22 & ++ \\
6 & M & 25 & 3 & 27 & + \\
7 & M & 24 & 8 & 62 & + \\
8 & M & 24 & 3 & 27 & + \\
9 & M & 22 & 4 & 50 & + \\
10 & M & 22 & 8 & 22 & + \\
11 & F & 18 & 6 & 38 & + \\
12 & M & 17 & 4 & 18 & + \\
13 & M & 17 & 4 & 17 & + \\
14 & F & 16 & 5 & 26 & + \\
\hline
\end{tabular}

*Patient with synovitis. $\mathrm{MCP}=$ metacarpophalangeal. 


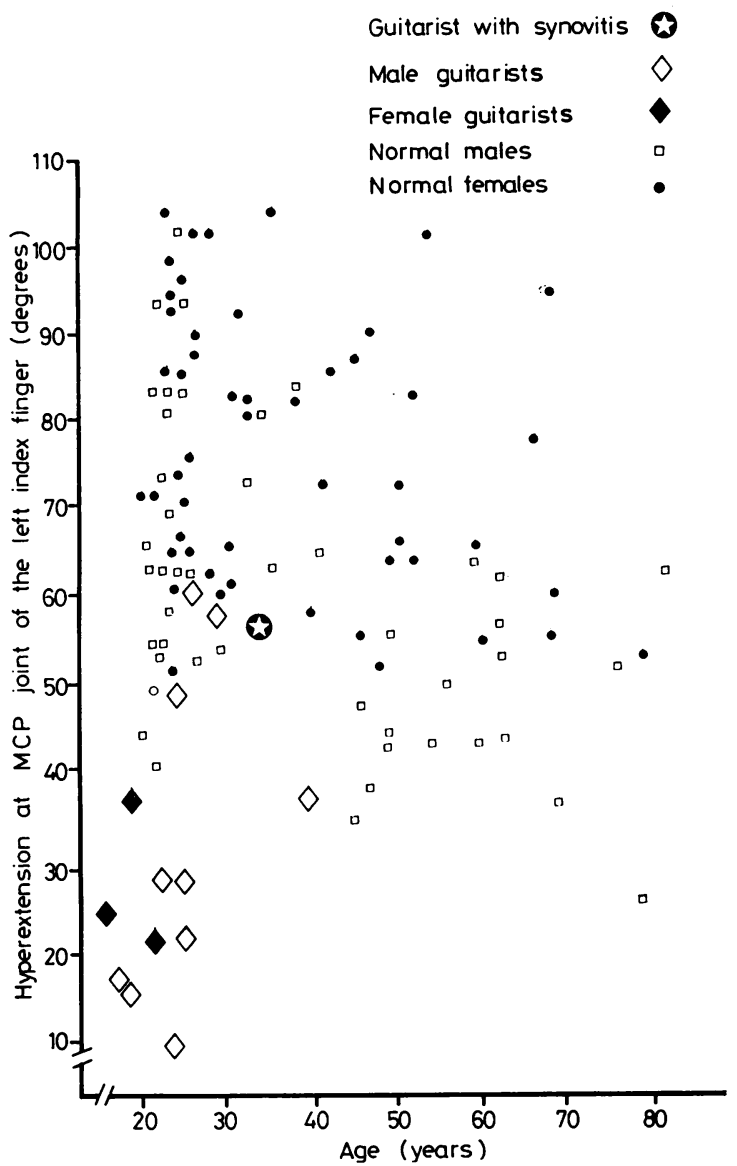

Fig. 4 Hyperextension at the metacarpophalangeal joint of the left index finger in 14 classical guitarists and 100 normal individuals.

reasonably classified as 'double-jointed' compared with other members of the group.

Studies with the finger hyperextensometer in a normal Caucasian population confirm a reduction in laxity at the metacarpophalangeal joint of the index finger of the left hand with age. Greater laxity is seen in females than in males of the same age (Fig. 4). Reduced laxity was a striking persistent finding in the guitarists, of either sex, compared with the normal population.

\section{Discussion}

We found no evidence that joint laxity increased in proportion to the number of years of guitar playing. The distribution of joint laxity within the guitar class resembled that of a normal population, though uniformly reduced. The torque adjustment of the hyperextensometer remained unaltered throughout the study, and the most likely explanation of the difference observed between the populations is that of greater muscular control in the fingers of guitar players as a result of regular practice. Increased muscular control has been shown to reduce the measured range of movement at other joints in the body in trained athletes. ${ }^{5}$ Since joint laxity measured in this study was independent of the duration of classical guitar training, it is possible that muscular control is acquired early and remains unaltered. However, a longitudinal study of novice guitar players over the first 6 months' training would be required to establish whether these individuals were selected because of joint laxity and subsequently stabilised their joints.

The guitarist with synovitis had strikingly lax hands, probably inherited from his father. The hand measured, the left, is that subjected to the greatest lateral spread in guitar playing, and it may be significant that his right hand was spared. This together with the serological results, clinical sparing of the metacarpophalangeal joints, and the lasting remission with steroid injection suggest he did not have rheumatoid arthritis in spite of the family history.

This study suggests that inherited factors may be more important than acquired in the aetiology of some forms of traumatic synovitis.

We thank the pupils and tutors at the Leeds College of Music for their co-operation in this study; Mr A. Moreton for technical services, and Mrs D. K. Smith for secretarial assistance.

\section{References}

1 Grahame R. Joint hypermobility-clinical aspects. Proc $R$ Soc Med 1971; 64: 692-4.

2 Bird H A, Tribe C R, Bacon P A. Joint hypermobility leading to osteoarthrosis and chondrocalcinosis. Ann Rheum Dis 1978; 37: 203-11.

3 Beighton P, Soloman L, Soskolne C L. Articular mobility in an African population. Ann Rheum Dis 1973; 32: 413-8.

4 Jobbins B, Bird H A, Wright V. A joint hyperextensometer for the quantification of joint laxity. Engineering in Medicine 1979; 8: 103-4.

5 Bird H A, Brodie D A, Wright V. Quantification of joint laxity. Rheumatol Rehabil 1979; 18: 161-6. 